\title{
LIFETIME CONTRIBUTION MEASUREMENTS AT THE AUSTRALIAN SYNCHROTRON
}

\author{
M.J. Spencer, M.J. Boland, R.T. Dowd, G. LeBlanc, Y.E. Tan, ASP, Melbourne, Australia
}

\section{Abstract}

There are always a number of factors that contribute to the lifetime of a stored particle beam. Measurements presented here show the relative importance of these effects during the commissioning of the Australian Synchrotron storage ring.

\section{INTRODUCTION AND THEORY}

In this paper we isolate and quantify a number of effects which contribute to the total beam lifetime at the Australian Synchrotron. These effects include gas scattering, intrabeam scattering, and the quantum lifetime. Gas scattering can be divided into elastic and inelastic scattering from both gas nuclei and electrons. The quantum lifetime will not be addressed as it does not contribute under normal circumstances. The total lifetime can be calculated using equation 1 [1].

$$
\frac{1}{\tau_{\text {total }}}=\frac{1}{\tau_{1}}+\frac{1}{\tau_{2}}+\ldots
$$

The individual lifetime effects are [1-3]:

Elastic scattering from gas nuclei:

$$
\frac{1}{\tau_{\text {nuclei-elastic }}}=\frac{2 \pi r_{e}^{2} c n Z^{2}}{\gamma^{2}} \frac{\bar{\beta} \beta_{a}}{a^{2}} .
$$

Inelastic scattering from gas nuclei:

$$
\frac{1}{\tau_{\text {nuclei-inelastic }}}=\frac{4 r_{e}^{2} c n Z^{2}}{137} \frac{4}{3} \ln \left(\frac{183}{Z^{1 / 3}}\right)\left(\ln \frac{1}{\Delta p / p}-\frac{5}{8}\right)
$$

Elastic scattering from gas electrons:

$$
\frac{1}{\tau_{\text {electron-elastic }}}=\frac{2 \pi r_{e}^{2} c n Z}{\gamma} \frac{1}{\Delta p / p}
$$

Inelastic scattering from gas electrons

$$
\frac{1}{\tau_{\text {electron-inelastic }}}=\frac{4 r_{e}^{2} c n Z}{137} \frac{4}{3} \ln \left(\frac{2.5 \gamma}{\Delta p / p}-1.4\right)\left(\ln \frac{1}{\Delta p / p}-\frac{5}{8}\right)
$$

Touscheck (intrabeam) scattering:

$$
\frac{1}{\tau_{T}}=\frac{r_{e}^{2} c N_{b}}{8 \pi \sigma_{x} \sigma_{y} \sigma_{l} \gamma^{2}} \frac{1}{(\Delta p / p)^{3}} D(\varepsilon)
$$

\begin{tabular}{|c|c|c|}
\hline$r_{e}$ & Electron radius & $2.8 \times 10^{-15} \mathrm{~m}$ \\
\hline $\bar{\beta}$ & $\begin{array}{l}\text { Average vertical } \\
\text { beta function }\end{array}$ & $14.6 \mathrm{~m}$ \\
\hline$\beta_{a}$ & $\begin{array}{l}\text { Vertical beta } \\
\text { function at } \\
\text { aperture }\end{array}$ & $\begin{array}{c}4.5 \mathrm{~m} \text { (at scraper)/ } \\
3.5 \mathrm{~m} \text { (nominal) }\end{array}$ \\
\hline$a$ & $\begin{array}{l}\text { Vertical half } \\
\text { aperture }\end{array}$ & $\begin{array}{c}\text { Variable/ } \\
5.5 \mathrm{~mm} \text { (nominal) }\end{array}$ \\
\hline$\gamma$ & Lorentz factor & 5871 \\
\hline$Z$ & $\begin{array}{c}\text { Residual gas } \\
\text { atomic number }\end{array}$ & 7 \\
\hline$c$ & Velocity of light & $3 \times 10^{8} \mathrm{~m} / \mathrm{s}$ \\
\hline$n$ & $\begin{array}{l}\text { Residual gas } \\
\text { density in units } \\
\text { of }\left(\mathrm{m}^{3}\right)\end{array}$ & $\begin{array}{c}\text { Approx: } \\
1.5 \times 10^{-9} \mathrm{mbar} \text { at } \\
100 \mathrm{~mA} \text {. This is } \\
3.68 \times 10^{13} \mathrm{~m}^{-3}\end{array}$ \\
\hline & Electrons per & $7.49 \times 10^{11}$ \\
\hline$N_{b}$ & & $\begin{array}{l}\text { (with } 50 \mathrm{~mA} \text { in } \\
300 \text { bunches) }\end{array}$ \\
\hline$\sigma_{x}$ & Bunch width & $\begin{array}{l}99.2 \text { um (at bend) } \\
459 \text { um (average) }\end{array}$ \\
\hline$\sigma_{y}$ & Bunch height & $\begin{array}{c}61.1 \text { um (at bend) } \\
41 \text { (average) }\end{array}$ \\
\hline$\sigma_{l}$ & Bunch length & $10 \mathrm{~mm}$ \\
\hline$\Delta p / p$ & $\begin{array}{l}\text { Momentum } \\
\text { acceptance } \\
\text { parameter }\end{array}$ & $\begin{array}{l}\text { Ill defined, } \\
0.01-0.03\end{array}$ \\
\hline$\beta_{x}$ & $\begin{array}{l}\text { Beta function at } \\
\text { the source point }\end{array}$ & $0.4 \mathrm{~m}-0.5 \mathrm{~m}$ \\
\hline
\end{tabular}

where $D$ is a numerically evaluated function [4] of $\mathcal{E}$.

Where $\varepsilon=\left(\frac{\Delta p / p \beta_{x}}{\gamma \sigma_{x}}\right)^{2}$. In our case $D \cong 0.25$
Table 1: List of variables, their meanings and values

The residual gas atomic number of 7 has been used in a number of studies by other similar rings [1-2]. Our residual gas analysers confirm a peak in the mass spectrum due to $\mathrm{CO}$ and $\mathrm{N}_{2}$. The average residual gas atomic number can reasonably be assumed to be 7 in our case.

The purpose of this study was to create circumstances in which a certain lifetime effect will dominate. Measurements of the total lifetime can then be used to confirm the values used in equations 2-6.

Since equations 3, 4 and 5 all simply depend on the gas pressure and the momentum acceptance then they are hard to isolate. Nuclei elastic scattering can be made dominant by decreasing the aperture, and Touscheck scattering can be made to dominate by increasing the bunch density through reduced coupling or a reduced number of bunches. 


\section{GAS SCATTERING}

\section{Gas Scattering Contribution}

It can be seen from equation 2-6 that all contributions to the lifetime will scale linearly with current. It can also be seen that the inverse Touscheck lifetime will reduce to zero as the current reduces to zero, however, the gas lifetimes will remain finite because there is a residual gas pressure when there is zero current.

In figure $1 \mathrm{a}$ plot is made of $1 /$ lifetime vs. current for three different values of coupling. Varying the coupling modifies the Touscheck contribution but will not change the gas scattering contributions. From the graph the yintercept is found to be approximately $2.8 \times 10^{-6} \mathrm{~s}^{-1}$ which corresponds to a lifetime of about $100 \pm 10 \mathrm{~h}$. Using equations 1-6 with a momentum acceptance of 0.015 and zero Touscheck contribution this implies a gas pressure of $3.6 \times 10^{-10}$ mbar. This is in close agreement with the measured vacuum pressure at zero current.

The beam began to display instabilities above $80 \mathrm{~mA}$ which had the effect of increasing the vertical beam size. At low coupling this had the effect of increasing the lifetime. The two points above $80 \mathrm{~mA}$ were not used in the lines of best fit.

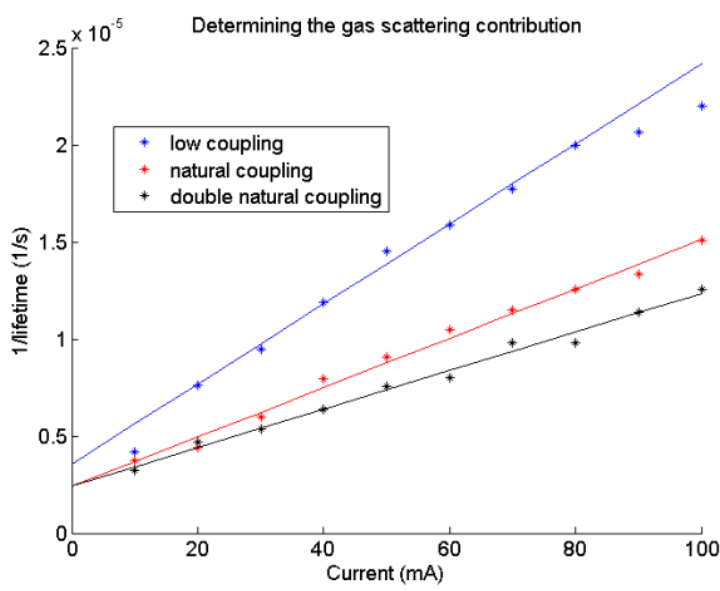

Figure 1: Inverse lifetime as a function of current.

\section{Elastic Scattering from Gas Nuclei}

Elastic scattering from gas nuclei excites betatron oscillations which can exceed the aperture. Equation 2 implies that as the aperture is reduced (using scrapers), the contribution from this effect will begin to dominate the total lifetime. Under these conditions $\frac{1}{\tau_{\text {total }}} \propto \frac{1}{a^{2}}$.

To reduce the Touscheck component data was obtained with a lower electron density by filling the ring with $50 \mathrm{~mA}$ in 300 buckets and increasing the coupling with skew quadrupoles.

Figure 2 shows 1/lifetime plotted as a function of $1 / \mathrm{a}^{2}$. While elastic scattering from gas nuclei dominates, the data should behave linearly, unfortunately, this is complicated by the fact that the beam current also decreases as the aperture is reduced the.

The theoretical gradient was plotted for a vacuum of $1.5 \times 10^{-9}$ mbar. The offset in the data of $3.5 \times 10^{-6} \mathrm{~s}^{-1}$ corresponds to a lifetime of $80 \pm 10$ hours. This can be assumed to be the sum of all other lifetime contributions.

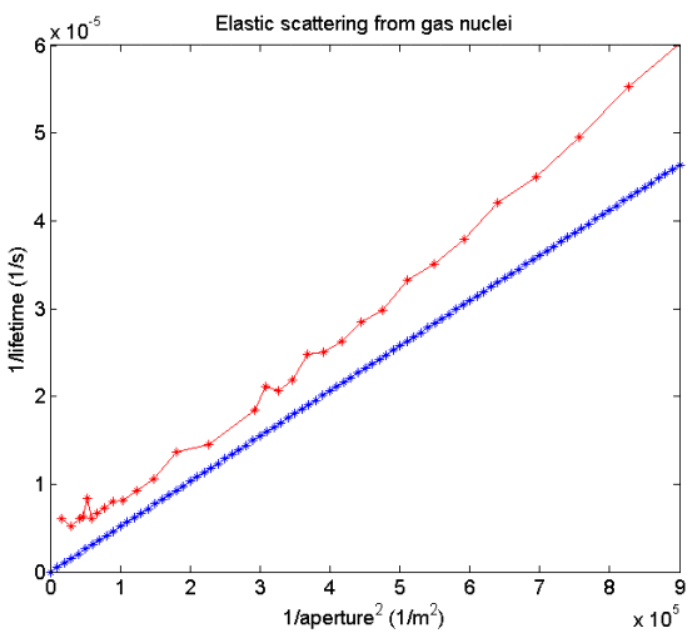

Figure 2: $\frac{1}{\tau_{C}}$ vs. $\frac{1}{a^{2}}$. The theoretical gradient is plotted with the experimental data.

\section{TOUSCHECK EFFECT}

With a high bunch current and reduced coupling it is possible to ensure that the Touscheck effect dominates the lifetime of the beam. Under these conditions the number of bunches was varied while keeping the current at a constant $10 \mathrm{~mA}$.

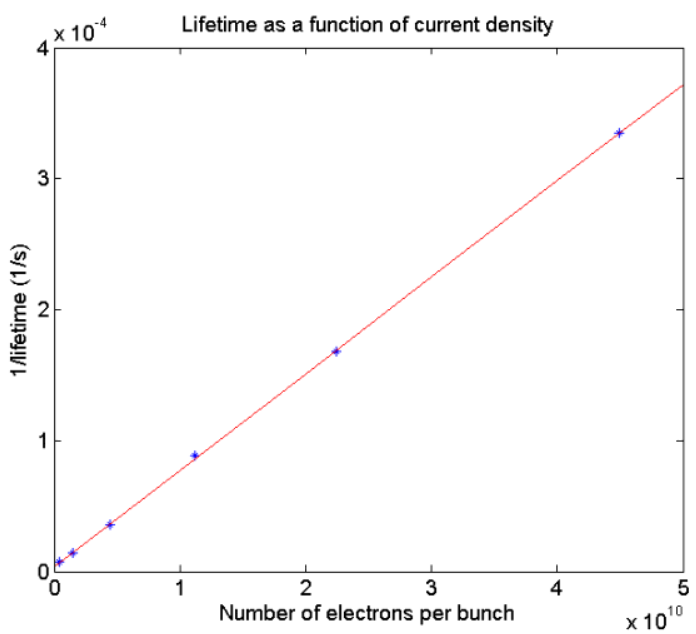

Figure 3: Lifetime as a function of current density.

The fit has a gradient of $7.36 \times 10^{-15} \mathrm{~s}^{-1}$ and an intercept of $3.73 \times 10^{-6} \mathrm{~s}^{-1}$. The intercept of the fit corresponds to a lifetime of $74.5 \mathrm{~h}$. If it is assumed that this value is the gas scattering contribution then with momentum acceptance of 0.015 equations $2-5$ imply that the vacuum must be $4.8 \times 10^{-10}$ mbar at $10 \mathrm{~mA}$. This is in accordance 
with the value found from figure 1 which was for zero current.

The gradient of the fit implies a much lower beam volume than was measured, or a lower than expected momentum acceptance. This is as yet un-resolved.

\section{INTEGRATED CURRENT}

\section{Overall Conditioning}

At the time of writing, the integrated beam current was $121 \mathrm{Ah}$. Over the course of the last year a number of straight section vacuum chambers have been replaced to allow for small gap insertion devices. It can be seen from figure 4 that these new vacuum chambers have not had a significant impact on the vacuum chamber conditioning process.

The storage ring RF system has not yet been run at full voltage. It is expected that increasing the cavity voltage will increase the energy acceptance and therefore the lifetime* current product.

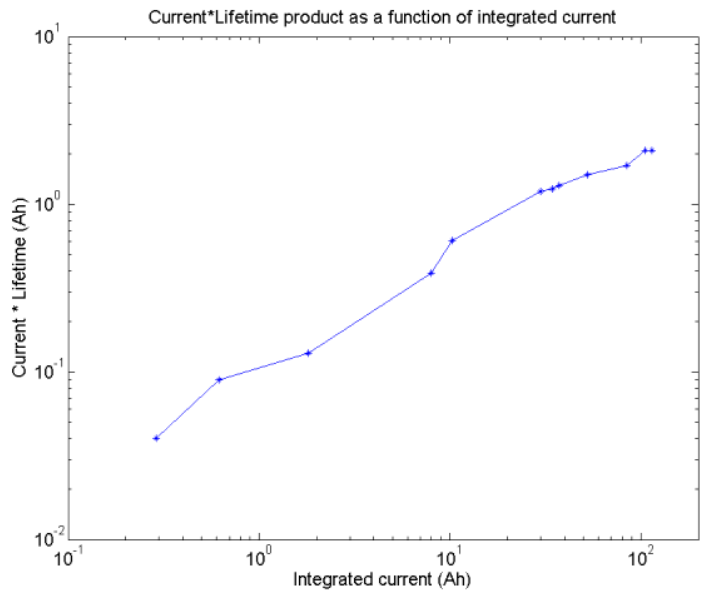

Figure 4: The lifetime current product as a function of integrated current.

The current-lifetime product has reached a value higher than $2 \mathrm{Ah}$. This indicates that with reduced coupling the ring lifetime should be dominated by the Touscheck effect.

\section{New Narrow Gap Chamber Conditioning}

At 94.5 Ah total integrated current a narrow gap insertion device was installed in the ring. It can be seen (fig 5) that 1 Ah was enough to restore the ring to its original currentlifetime value.

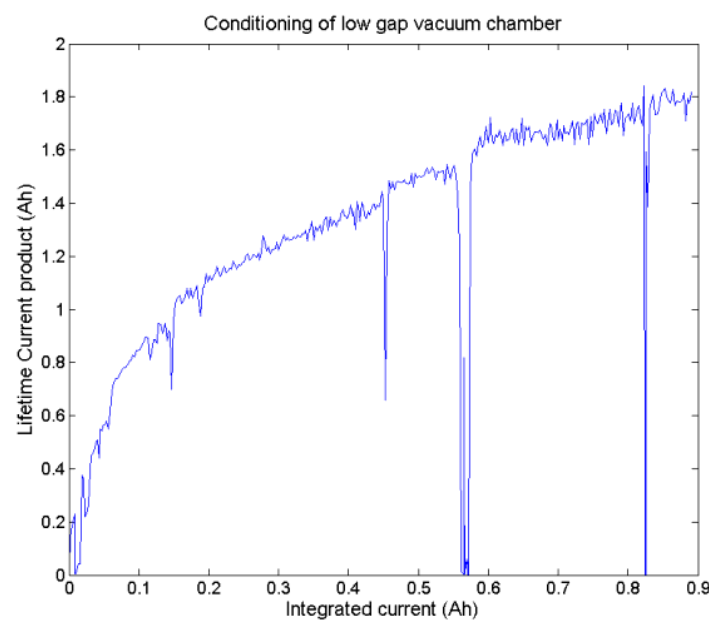

Figure 5: The period after a narrow gap chamber was installed in one of the straights. Before installation the current-lifetime product was $1.8 \mathrm{Ah}$ and the integrated current was $94.5 \mathrm{Ah}$.

\section{DISCUSSION AND CONCLUSION}

By undertaking a study of the lifetime contributions it was possible to narrow down the values for certain machine parameters. In particular, the accelerator group has increased their understanding of the vacuum pressure, momentum acceptance, and bunch volume.

Most pressures in the ring increased by a factor of 3 as the beam current was increased to $100 \mathrm{~mA}$ while others increased by up to a factor of 10 . It was difficult to calculate an average vacuum pressure under these circumstances.

Since the Australian Synchrotron storage ring is in the late stages of commissioning, changes to the nominal operating values of the RF voltage and the vacuum pressure are still occurring. It will be beneficial to repeat the lifetime study outlined above, once the operating parameters have become more stable.

\section{REFERENCES}

[1] E.Huttel, I.Birkel, A.-S.Müller, F.Perez, M.Pont, "Studies of beam lifetime at ANKA", PAC'03, p. 893.

[2] S. Khan, "Study of the Bessy II beam lifetime", PAC'99, New York 1999, p. 2831.

[3] Hana M. Bizek, "Some results of the Advanced Photon Source beam lifetime studies", PAC'97, Vancouver 1997, p. 1493.

[4] H. Wiedemann, "Particle Accelerator Physics I", second edition, Springer, 2003. 